\title{
THE WEAKEST LINK OF A CHAIN DETERMINES ITS STRENGTH: VALUING THE PASSENGER RISK OF MISSED TRANSFERS
}

\author{
MICHAEL BUNDSCHUH ${ }^{1}$, MICHAEL LEFELD ${ }^{2}$, JOHANNES LIEBERHERR $^{3} \&$ KLAUS NOEKEL $^{1}$ \\ ${ }^{1}$ PTV AG, Germany \\ ${ }^{2}$ Deutsche Bahn AG, Germany \\ ${ }^{3}$ ttools gmbh, Switzerland
}

\begin{abstract}
Reliable transfers are an essential characteristic of successful timetables, as passengers expect seamless door-to-door mobility. Yet most current travel demand models used for strategic timetable design do not support the evaluation of transfer reliability. An indicator is proposed which values the risk of missed transfers as the expected additional door-to-door travel time. The indicator allows transfers to be classified according to the total damage which breaking it would cause to passengers' travel plans, in terms of later arrival at the final destination, and not just the extra wait time at the transfer station. The definition, calculation method, and data sources are discussed in turn. The most immediate application of the indicator is to identify high-risk transfers during strategic timetable design and apply multi-criteria comparison between timetable variants including the transfer risk in addition to more conventional indicators like travel time or number of transfers. Several additional use cases are presented, ranging from macro-economic valuation of passenger time loss within cost-benefit analyses, to estimating the amount of penalty payments by operators to passengers due to large delays, and assessing the value of infrastructure improvements from the perspective of reducing passenger risk.
\end{abstract}

Keywords: timetable construction, risk management, passenger demand models, delays, passenger transfers.

\section{INTRODUCTION}

Many railway operators employ a tiered approach to timetabling: at the strategic level a timetable is optimized to match passenger demand, and this timetable is then refined towards the operational timetable. At the strategic level travel demand models [1]-[3] are used to forecast level of service for several timetable scenarios, and the demand reaction to those. In order to allow many scenarios to be evaluated, these models make several simplifying assumptions. One is that the planned timetable is deterministically executed without delays. Passengers can therefore plan their journeys pre-trip and can expect the execution of their travel plan with certainty. The traffic assignment models capture passenger behaviour by searching several connections for each origin-destination pair, computing the (dis)utility of each, and splitting the total demand across these choices according to utility. Typically, the utility function $U$ (conn) for a connection conn is formulated as a linear combination of various travel time components and monetary cost, as exemplified in eqn (1).

$$
U=\beta_{\text {veh }} t_{v e h}+\beta_{a c c} t_{\text {acc }}+\beta_{\text {walk }} t_{\text {walk }}+\beta_{\text {wait }} t_{\text {wait }}+\beta_{\text {fare }} F+\beta_{\text {trans }} n_{\text {trans }},
$$

where $t_{v e h}, t_{\text {acc }}, t_{\text {wait }}, t_{\text {walk }}$ are in-vehicle, access/egress, waiting, and walk time, $F$ is fare, $n_{\text {trans }}$ is the number of transfers, and $\beta_{i}<0$ are coefficients expressing passenger preferences. The a priori chosen connections are called planned connections.

In real operation stochastic disturbances induce delays and the actual arrival time can be later than in the planned connection. If a journey involves transfers, arrival at the final destination may be much later than scheduled, because a transfer breaks and the passenger 
has to wait for the next available connection, often by an hour or more. The connection to which the passenger switches en-route is called the alternative connection.

[4] analyzes the total social cost of timetable disruption and distinguish several cost categories, including extended travel times due to unreliability and uncertainty, and the cost of taking evasive action. For the Netherlands alone, the authors estimate the two categories at 200-300 million Euros per year. Where the probability of missed transfers is so high that passengers become aware of it, it may influence route choice and consequently even mode choice.

Although these effects should therefore be considered during strategic timetabling, most travel demand models ignore them. On the contrary, if models based on utility functions like eqn (1) are used to compare timetable scenarios, then all other things being equal, timetables with short in-vehicle times and transfer waiting times are favoured because they maximize passenger utility. These timetables look good on paper but are often not robust against stochastic disturbances. Because buffer times are minimized, even small original delays will break transfers and extend passenger travel times significantly.

The objective of this work is to define an indicator which captures the user perception of the risk of missing a transfer and which can be added as an additional term to eqn (1). It counterbalances the travel time terms, because the risk of delays and of knock-on effects tends to increase in streamlined timetables, and so would the passenger risk of missing transfers. In real operations predicting the actual arrival time at the final destination is complex and depends on aspects like delay propagation along the line and dispatching decisions. Since the objective here is to measure passenger risk, not dispatching quality, and to develop a computationally feasible method, two simplifying assumptions are made:

- After a train experiences an initial delay, this delay remains constant until its destination stop.

- Within the analysis of the effects of one delayed train, all other trains are assumed to run according to the planned timetable.

This paper builds on [5]. It differs from the earlier work in several aspects:

- The situations in which passengers are assumed to take evasive action if a transfer breaks, more closely match those where the behaviour would be expected in reality.

- Assumed input data, particularly on original delay probabilities, more closely match data actually available.

- Several real-world applications are reported.

\section{DEFINITIONS AND MODEL}

The definition of the indicator valuing additional door-to-door travel time caused by delays generally follows concepts developed in [5], but with some modifications. Compared to the earlier paper, the definition of delays is considerably changed and focuses on the special situation that passengers receive information about delays of the run they currently travel on just when reaching the planned transfer stop.

\subsection{Supply}

The setting can be summarized as follows: Let $G=(N, R S, T T)$ be a multi-graph modeling the transit supply, where the set of nodes $N$ represents public transport stops. Each train service is called a run. Each edge in set $R S$ represents a run segment, i.e. a piece of a run 
between successive stops. Each edge $r s=(u, v$, dep, arr $)$ from source node $u$ to target node $v$ is labeled with a (planned) departure time dep at $u$ and a (planned) arrival time arr at $v$, such that $\operatorname{arr}>\operatorname{dep}$. Multiple edges may connect a pair of nodes $u, v$ in $N$.

An edge path $P \subset R S$ is called time-consistent, iff for any two successive edges $r s_{1}, r s_{2} \in$ $P: \operatorname{arr}\left(r s_{1}\right) \leq \operatorname{dep}\left(r s_{2}\right)$. TT (the "timetable") is the set of runs. Each run is a set of run segments which forms a time-consistent path in $G$.

For each run segment rs $\Delta_{r s}$ denotes the distribution function describing the delay distribution of the arrival of $r s$ at $v$ and $\delta_{r s}$ its density, i.e.

$$
\Delta_{r s}(x)=P\left(a r r_{\text {actual }}(r s)-\operatorname{arr}(r s) \leq x\right)=\int_{0}^{x} \delta_{r s}(t) d t
$$

Note that $\Delta_{r s}(0)$ is the probability of $r s$ arriving on time or earlier. There is no distinction between original and propagated delays. In applications one can therefore directly use the observed (or estimated) delay distribution for each arrival in the timetable.

\subsection{Demand}

Since the aim is to evaluate additional travel time from the passengers' point of view, demand must be defined. A connection conn is a time-consistent path $P \subset G$ from an origin stop $O$ to a destination stop $D$ used by a passenger volume vol(conn). Successive edges of $P$ belonging to the same run $r \in T T$ form a leg. Legs are separated by transfers between different runs. The demand is the set of all planned connections conn together with their respective volume $\operatorname{vol}($ conn).

Connections can be the output of a schedule-based assignment which loads trips from an OD trip table onto the public transport network $G$, but it can also be any set of observed passenger data from surveys or electronic ticketing devices.

\subsection{Risk of extended travel time}

\subsubsection{Basic definition}

At the most detailed level, the expected additional travel time can be defined for one particular connection conn and one particular transfer at node $t$ on this connection. Let $r s_{0}$ be the last run segment of conn before $t$, i.e. the target node of $r s_{0}$ is $t$, and let the arrival of $r s_{0}$ be delayed by $\delta_{a r r}$. In this situation the whole run $r_{0}$ of $r s_{0}$ is shifted in time by $\delta_{a r r}$, i.e. by adding in the graph $G \delta_{\text {arr }}$ to the departures and arrivals of all segments belonging to the same run as $r s_{0}$, and then searching for reasonable alternative connections from $r s_{0}$ to the final destination $D$ of the initial connection conn as reaction to the modified situation. This includes the option of staying on board of the run $r_{0}$ at $t$, assuming its delay will remain unchanged until its destination.

Searching 'reasonable' alternative connections is analogous to searching planned connections with the original assignment, including the notion of utility as in eqn (1), but with the delayed run segment $r s_{0}$ as new origin and volume vol(conn) instead of total demand on O-D. As within the assignment, branch\&bound search produces a choice set of alternative connections from $r s_{0}$ to destination $D$. Total demand vol(conn) is distributed across the different alternatives based on their utility. Any alternative connection $c$ ' for planned connection conn has its own arrival time $\operatorname{arr}\left(c^{\prime}\right)$, which could be earlier than $\operatorname{arr}($ conn) possibly because the definition of utility is different from the definition used during initial assignment. Since the proposed indicator should measure the increase of travel time due to 
breaking transfers, not the quality of the planned or observed connection, only the positive part $\max \left(\operatorname{arr}\left(c^{\prime}\right)-\operatorname{arr}(\operatorname{conn}), 0\right)$ of the difference between alternative and planned connection is taken. The weighted average of these delays per alternative the average delay at destination $\operatorname{DaD} D_{\text {conn }, t}\left(\delta_{\text {arr }}\right)$ given delay $\delta_{\text {arr }}$.

By construction, DaD $D_{\text {conn }, t}$ is a function $\operatorname{DaD}_{\text {conn }, t}\left(\delta_{\text {arr }}\right): \mathbb{R}^{\geq 0} \rightarrow \geq \mathbb{R}^{\geq 0}$. Define the risk per passenger of connection conn at transfer $t$ to be its integral including the delay distribution:

$$
R_{\text {conn }, t}^{T}:=\int_{0}^{T} \operatorname{DaD_{\text {conn},t}}(x) \delta(x) d x .
$$

In principle, one could set $T$ to infinity (at least if the integral still has a finite value then), but this makes no sense in our approach: If the delay $\delta_{\text {arr }}$ is getting large, it is very likely that the assumption on all the other runs running on schedule does not hold true. On the other hand, large delays (hopefully) are rare exceptions, and hence do not contribute to the integral significantly. If there is something like a maximum headway in the supply, it is a good choice to take this as value for threshold $T$. If the delay distribution is rapidly falling on large values, threshold $T$ can be reduced to save calculation effort without losing too much of the skim's significance.

The case that the alternative connection arrives earlier than the planned one occurs in practice if the faster part of supply cannot be used by the passenger due to fare restrictions which are lifted in case of disruption. Since the passenger cannot rely on the disruption (causing an earlier arrival), this does not count as "negative extended journey time" - that is why only the positive part of travel time increase is used in the definition.

\subsubsection{Aggregated values}

So far, the definition of the risk of extended travel time per passenger $R_{c o n n, t}^{T}$ refers to one connection conn, one transfer $t$ on conn and threshold $T$. There are vol(conn) passengers experiencing this extended travel time at $t$, traveling on conn, but other connections may also include a transfer between the same run segments $r s_{0}$ and $r s_{l}$ at $t$. Summing up all these experienced travel time increases makes up the inherent risk of this transfer:

$$
\tilde{R}_{r s_{0}, r s_{1}, t}^{T}:=\sum_{\text {conn overrs } s_{0}, r s_{1} \text { at } t} R_{\text {conn }, t}^{T} \operatorname{vol}(\mathrm{conn}) .
$$

Note that this skim is not a risk per person, but the total perceived risk of all passengers using transfer $\left(r s_{0}, r s_{1}, t\right)$. For consistency, one can define an average risk per person

$$
R_{r s_{0}, r s_{1}, t}^{T}:=\frac{\tilde{R}_{r s_{1}, r s_{2}, t}}{\sum_{\text {conn }, t} \operatorname{vol}(\operatorname{conn})}
$$

Late arrival at the final destination dest can be included in the total risk of connection conn, by regarding the arrival at destination as an additional 'transfer' at dest. In this case there is only the trivial alternative connection, yielding $\operatorname{DaD}_{\text {conn,dest }}(x)=x$ and therefore $R_{\text {conn, dest }}^{T}=\int_{0}^{T} x \delta(x) d x=E^{T}(\Delta)$. Let 


$$
R_{\text {conn }}^{T}:=\sum_{\begin{array}{c}
\text { t transfer on conn } \\
\text { (including dest) }
\end{array}} R_{\text {conn }, t}^{T}
$$

be the risk of connection conn per person and

$$
\tilde{R}_{\text {conn }}^{T}:=\sum_{\begin{array}{c}
\text { transfer on conn } \\
\text { (including dest })
\end{array}} \tilde{R}_{\text {conn }, t}^{T}=R_{\text {conn }}^{T} \operatorname{vol}(\text { conn })
$$

the total risk of connection conn.

Summing up the risks of all transfers yields a network wide skim, whereas summing over suitable subsets of transfers yields risk assessments that can be viewed as the risk "caused by" certain parts of supply - e.g. summing over all transfers where the arriving run segment is operated by a certain operator or belongs to a certain class of runs (a certain "product").

\subsubsection{Calculating the risk of extended travel time}

It is important to note that the "delay at destination" function $D a D_{c o n n, t}$ is a step function: Increasing delay $\delta_{a r r}$ does not affect $\operatorname{Da} D_{\text {conn }, t}\left(\delta_{\text {arr }}\right)$ as long as the same set of alternative connections can still be realized. In other words: $\operatorname{Da} D_{\text {conn,t }}(x)$ is constant unless $x$ is in the finite set of values $\left\{x_{1}, \ldots, x_{m}\right\}$ where any transfer originating from $r s_{0}$ at $t$ (not necessarily the transfer incorporated in the initially planned connection conn) is missed. That means, it is possible to look up these $x_{i}$ efficiently and a priori from the timetable, and hence calculating these risks involves a finite set of searches for alternative connections per transfer and connection.

The computational cost for searching for alternative connections remains nearly the same when adding additional destinations, and the set of time points $x_{i}$ where any transfer originating from $r s_{0}$ breaks does not depend on the connection conn. Therefore, it is convenient to search for the alternative connections and though to calculate the functions $D a D_{\text {conn,t }}$ for all connections conn having any transfer following run segment $r s_{0}$ in one step. Note that since the DaDs are step functions, the integral defining the risk can be evaluated piecewise, thus a density function of the distribution is not really needed:

$$
\begin{aligned}
R_{\text {conn }, t}^{T}= & \sum_{x_{i} \in\left\{x_{1}, \ldots, x_{m}, T\right\}} \int_{x_{i-1}}^{x_{i}} \operatorname{DaD}_{\text {conn }, t}(x) \delta(x) d x \\
& =\sum_{x_{i} \in\left\{x_{1}, \ldots, x_{m}, T\right\}} \operatorname{DaD}_{\text {conn }, t}\left(x_{i}\right) P\left(x_{i-1}<x \leq x_{i}\right) .
\end{aligned}
$$

In applications this means that it is possible to use observed statistical data directly.

\subsubsection{Passenger distributions}

This internal nature of the proposed skims can be exploited in another way: the calculation of delay at destination for a certain delay situation of an arriving run segment $r s_{0}$ involves searching for alternative connections and distributing the passengers traveling on affected connections onto these alternative connections. Let $d_{i}$ denote this distribution with $\sum d_{i}=1$. In this situation each alternative connection has its fixed arrival time at destination, gets a defined portion $d_{i}$ of the initial connection's volume, and the current delay situation together 
with all its equivalents has probability given by $P\left(x_{i-1}<x \leq x_{i}\right)$. Summing up the volume of all alternatives with arrival time falling into given intervals, but belonging to different delay situations yields the absolute number of passengers experiencing delays in corresponding intervals:

$$
A_{\text {conn }}\left(y_{i-1}, y_{i}\right)=\sum_{\begin{array}{c}
\text { alt is any alternative for conn } \\
\text { with arrival in } \left.] y_{i-1}, y_{i}\right] \\
\text { if delay situation in } \left.] x_{t_{0}-1}, x_{t_{0}}\right]
\end{array}} d_{\text {alt }} \operatorname{vol}(\text { conn }) P\left(x_{t_{0}-1}<x \leq x_{t_{0}}\right) .
$$

More generally speaking, in addition to the expected extended travel time the proposed method yields the distribution of experienced delays.

\section{APPLICATIONS}

The risk indicators defined in Section 2 can be used in various stages of the strategic timetabling process. This section describes several applications and reports initial results. All figures shown are based on fictitious or modelled data.

The first application was already motivated in Section 1. Travel demand models ignore the effect of risk on passenger connection choice. Traffic assignment should yield more realistic choice proportions, if the utility function from eqn (1) were replaced by

$$
\begin{aligned}
& U^{\prime}=\beta_{\text {veh }} t_{\text {veh }}+\beta_{\text {acc }} t_{\text {acc }}+\beta_{\text {walk }} t_{\text {walk }}+\beta_{\text {wait }} t_{\text {wait }}+\beta_{\text {fare }} F+ \\
& \beta_{\text {trans }} n_{\text {trans }}+\beta_{\text {risk }} R_{\text {conn }}^{T},
\end{aligned}
$$

where the risk indicator is summed over all transfers nodes of the planned connection conn. Interestingly, more literature exists on estimating the value of travel time reliability, very similar to $\beta_{\text {risk }}$, from surveys [6] and [7] than on methods for quantifying the (un)reliability in traffic assignment.

An example illustrates the significance of the risk term in the (dis)utility of connections: on the OD pair Koblenz Hbf - Leipzig Hbf every hour there is a connection with one transfer. In even hours the transfer takes place at Frankfurt a.M. Flughafen (airport), in odd hours the transfer takes place at Mainz Hbf. Without consideration of transfer risk, the two connections are comparable (in term of the disutility $U$ ). But the transfer in Mainz Hbf is very risky: first, the transfer buffer time is $3 \mathrm{~min}$ (after deduction of 2 min transfer/walk time); second if the transfer is broken, the next direct and fast alternative connection from Mainz Hbf to Leipzig $\mathrm{Hbf}$ is two hours later. Hence the resulting delay in Leipzig Hbf in case of a broken transfer in Mainz Hbf is almost two hours. The transfer of the other connection in Frankfurt a.M. Flughafen is - due to a buffer time of $10 \mathrm{~min}$ - much less risky. This leads to the fact that the disutility of the second connection in Fig. 1 is significantly higher than of the first connection.

While the utility function in eqn (10) leads to more realistic connection choice in the presence of risky transfers, one objective of strategic timetabling should be to eliminate the most severe cases from the planned timetable in the first place. The challenge is to identify these cases, especially as traditionally predicted numbers of passengers using each run or transfer are not used during timetable construction.

The timetable designer can rank all potential transfers in the timetable by risk indicator and systematically work on those with highest risk, caused either by a high number of passengers using it or by a large travel time increase if it breaks. Since the risk indicator is calculated per transfer and connection (the smallest unit in macroscopic demand models for 


\section{Connection 1}

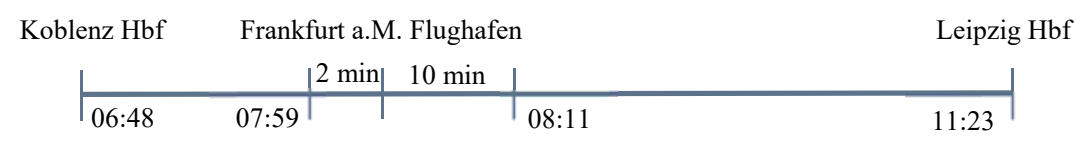

\section{Connection 2}

Koblenz Hbf
\begin{tabular}{|lr|r|r|r|} 
& Mainz Hbf & Leipzig Hbf \\
\hline $07: 48$ & $08: 38$ & $2 \mathrm{~min}$ & $3 \mathrm{~min}$ & \\
\hline
\end{tabular}

\begin{tabular}{|l|c|c|c|c|c|c|c|}
\hline & $t_{\text {veh }}$ & $t_{\text {walk }}$ & $t_{\text {wait }}$ & $n_{\text {trans }}$ & $R_{\text {conn }}^{T}$ & $U$ & $U^{\prime}$ \\
\hline Connection 1 & $04: 23$ & $00: 02$ & $00: 10$ & 1 & $00: 07$ & $04: 56$ & $05: 03$ \\
\hline Connection 2 & $04: 29$ & $00: 02$ & $00: 03$ & 1 & $00: 46$ & $04: 55$ & $05: 41$ \\
\hline
\end{tabular}

Figure 1: Example of two connections with different risk on the OD pair Koblenz Hbf Leipzig Hbf.

public transport), the analysis can be done at any desired level, just by aggregating the data, i.e. with a few mouse clicks. Typically, the analysis starts with a network plot which shows the average or total transfer risk per station. Stations with conspicuous transfer-risk can now be analyzed in more detail. Fig. 2 illustrates this top-down approach.

High risk can be mitigated in different ways:

- increasing the transfer time by some buffer,

- improving timekeeping upstream of the transfer node, thereby lowering the initial delay probability, or

- guaranteeing the transfer through dispatching, e.g. to have the connect train wait for some minutes.

The results of the first two measures will be reflected in the proposed risk indicator and can be compared directly to possible adverse consequences of the changes at other transfer nodes.

The idea of reducing risk by improving operational stability upstream of the transfer node can be developed further into a methodology for evaluating infrastructure improvements from a new perspective. Proposed investment into e.g. additional point work to reduce pathing conflicts is often justified exclusively in terms of improved operational stability, measured by indicators such as the reduction in the variance of running times. The reduction of the transfer risk for passengers can be an alternative customer-oriented return on investment (see for example [8]). 

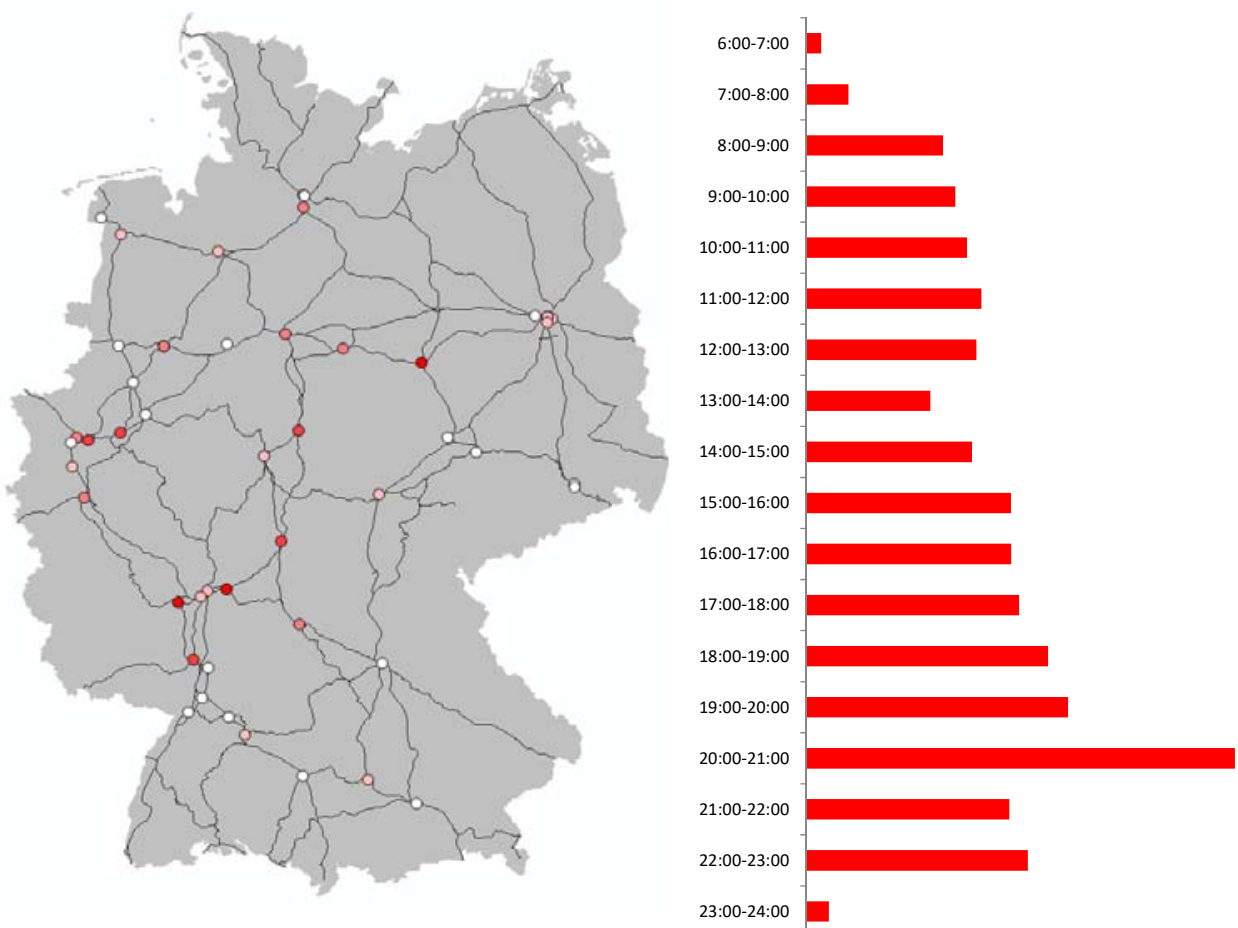



Figure 2: Different aggregation levels of risk analysis: network-wide (top left); per hour and station (top right); per train-to-train-transfer (bottom). 
Similar to this customer-oriented evaluation of infrastructure measures, the approach could also be applied to software-based solutions to improve the operational stability, e.g. algorithmic solutions which calculate the optimal speed per train or even self-driven trains.

Note that the methodology presented here always presupposes that the operational (un)punctuality, i.e. the delay distributions $\Delta_{r s}$ for each run segment $r s$ (see 2.1) are known, either from real data or from results of macroscopic or microscopic operation simulation.

In addition to the more infrastructure-oriented stabilization measures described so far, punctuality improvements are often also achieved by means of classical timetable planning adjustments such as reducing the number of trains on critical tracks, breaking lines at intermediate stops, omission of stops, moving buffer times from less critical to more critical sections, use of other rolling stock, or similar.

Consider an example: The longer the route of a line, the greater the delay. Breaking a line $L_{R e f}$ into two separate parts $L_{V a r, 1}$ and $L_{V a r, 2}$ at an intermediate station $S$ (see Fig. 3) has two competing consequences: On the one hand, the new line $L_{V a r, 2}$ on the second part can start on time in $S$ and does not suffer from delays from the first part $\left(L_{V a r, 1}\right)$ : for all passengers boarding in or after $S$ this is positive: passengers which only travel on the second part have more punctual arrivals at their destinations (risk of delay at destination) and passengers which transfer from the second part to other trains have safer transfers. On the other hand, for the passengers passing on $L_{R e f}$ through $S$, the impact is negative: they have to choose another connection with an additional risky transfer. The approach proposed in this paper allows to determine the entire net effect of such a stability measure in detail. Fig. 3 applies this usecase to a concrete example: the intercity line from Hamburg Hbf to Stuttgart Hbf/Basel SBB is broken in Köln Hbf into two lines (Ref denotes the reference case where the line $L_{R e f}$ runs the entire route from Hamburg Hbf to Stuttgart $\mathrm{Hbf} / \mathrm{Basel} \mathrm{SBB}, \operatorname{Var}$ denotes the variant case, where the line is broken into two new lines $L_{V a r, 1}$ and $L_{V a r, 2}$ in Köln Hbf). The rest of the timetable remains unchanged. In $\operatorname{Var}$ transfers from $L_{V a r, 1}$ to $L_{V a r, 2}$ and vice versa are possible (but risky).

Fig. 3(b) shows for north-south direction of the line on the one hand the significant increase of the risk of delay caused by transfers in Köln Hbf (due to the additional risky transfers). On the other hand, for stops after Köln Hbf, both the risk of delay caused by transfers and the risk of delay at destination (risk of delay of passengers who reach her destination) are significantly reduced. For the other direction (south-north) the effect is symmetric in Köln Hbf.

An effect to be taken into account is that some passengers are switching to other connections/lines due to the additional change in Köln Hbf. As a result, part of the risk of delay caused by transfers from the line under consideration is shifted to other lines.

In total (over the entire net) the risk of delay caused by transfers increases and the risk of delay at destination decreases. If both effects (delay caused by transfers and delay at destination) are added, the variant case performs better (in terms of risk of delay).

This positive effect on the risk of delay can now be contrasted with the negative impact on the quality of supply through the additional transfer in Köln $\mathrm{Hbf}$ and the associated reduction in passenger-kilometres, such that the proposed measure can be assessed in its entirety. 


\begin{tabular}{|c|c|c|c|c|}
\hline \multirow{2}{*}{ Station } & \multirow{2}{*}{ Code } & \multirow{2}{*}{$\frac{\text { Ref }}{L_{\text {Ref }}}$} & \multicolumn{2}{|c|}{ Var } \\
\hline & & & $\mathrm{L}_{\mathrm{Var}, 1}$ & $L_{\text {Var, } 2}$ \\
\hline Hamburg Hbf & $\mathrm{AH}$ & 1 & 1 & \\
\hline Hamburg-Harburg & AHAR & I & 1 & \\
\hline Bremen Hbf & $\mathrm{HB}$ & 1 & 1 & \\
\hline Osnabrück Hbf (oben) & $\mathrm{HO} \mathrm{O}$ & 1 & 1 & \\
\hline Münster(Westf)Hbf & EMSTP & I & 1 & \\
\hline Dortmund $\mathrm{Hbf}$ & EDO & 1 & 1 & \\
\hline Bochum Hbf & EBO & 1 & 1 & \\
\hline Essen $\mathrm{Hbf}$ & $\mathrm{EE}$ & 1 & 1 & \\
\hline Duisburg Hbf & EDG & 1 & 1 & \\
\hline Düsseldorf Hbf & KD & 1 & 1 & \\
\hline Köln Hbf & KK & 1 & 1 & 1 \\
\hline Bonn Hbf & KB & 1 & & 1 \\
\hline Koblenz Hbf & KKO & 1 & & 1 \\
\hline Mainz $\mathrm{Hbf}$ & FMZ & 1 & & 1 \\
\hline Mannheim Hbf & RM & 1 & & 1 \\
\hline Heidelberg Hbf & $\mathrm{RH}$ & I & & 1 \\
\hline \begin{tabular}{|l} 
Stuttgart $\mathrm{Hbf}$ \\
\end{tabular} & TS & 1 & & 1 \\
\hline Karlsruhe $\mathrm{Hbf}$ & RK & 1 & & 1 \\
\hline Freiburg(Brsg)Hbf & $\mathrm{RF}$ & 1 & & 1 \\
\hline Basel SBB & XSB & 1 & & 1 \\
\hline
\end{tabular}

(a)

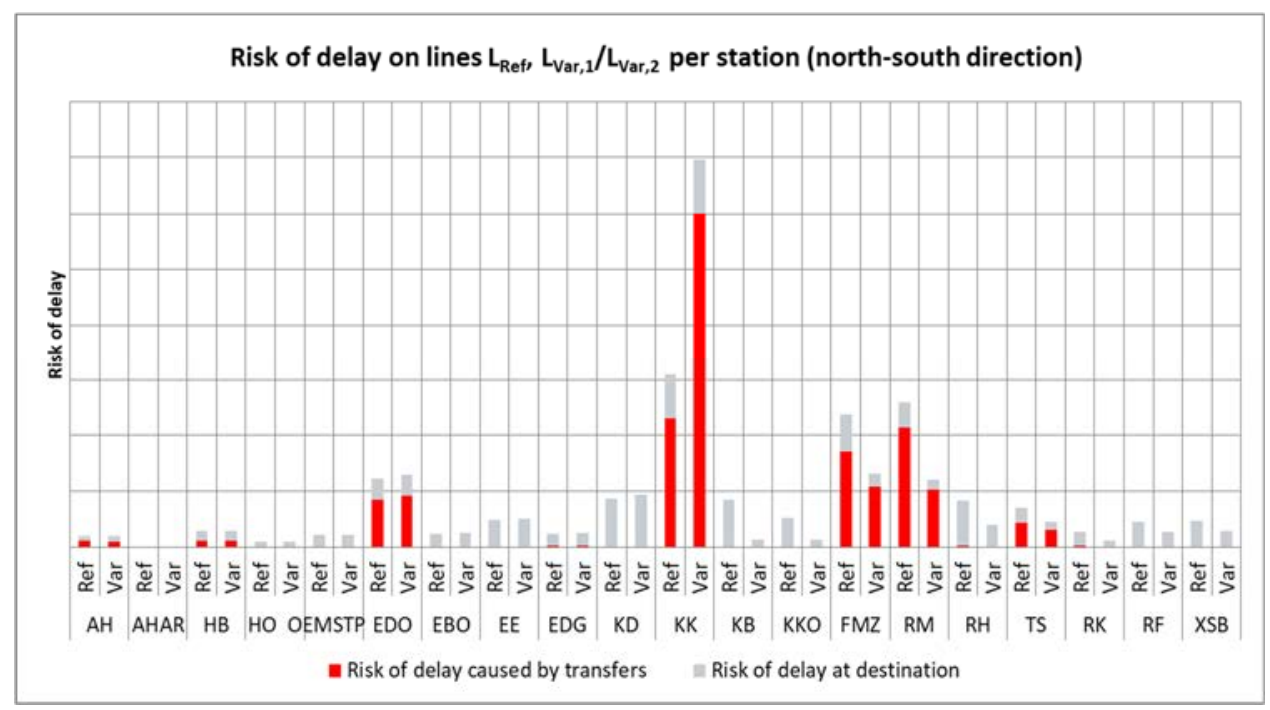

(b)

Figure 3: Consequences on risk of delay of the measure to break the intercity line Hamburg Hbf - Stuttgart Hbf/Basel SBB (Ref) in two new lines in Köln Hbf (Var). 


\section{CONCLUSION}

A new indicator is proposed which values the risk of passengers missing transfers in multileg travel plans. Compared to previous work [5] the definitions are adapted to correspond better to real-world decision situations. At the same time, the new indicator can be efficiently computed. In fact, the value of the proposal partly rests on the fact that the indicator is being implemented in the software package PTV Visum, widely used for strategic network and timetable development, so can be effectively applied in practice.

Applications range from more realistic travel demand forecasts via improving the robustness in timetables to assessing more comprehensively how infrastructure investments improve operational stability and to what extent this will benefit passengers.

Further work is planned both to extend the range of applications and to increase the realism of the method.

The new indicator could be used to forecast for future timetables some elements of social cost of service disruptions, thus complementing the empirical work in [4]. Another application could be based on the distributions of delays derived in section 2.3.4 from the proportion (or number) of passengers experiencing delays larger than a given threshold it is possible to calculate the total number of people who could claim their passenger rights, and the expected amount of compensation payments.

One possible improvement of realism concerns the assumption that only the subject train is delayed whereas all others run on time. Dispatchers may dynamically hold important connecting trains, which will affect the expected delays both for transferring passengers and for passengers on board the held train. The challenge is to treat the case in a way which improves realism and at the same time avoids combinatorial explosion in the calculation of the risk indicator.

\section{REFERENCES}

[1] Ortuzar, J. de D. \& Willumsen, L.G., Modelling Transport, Wiley, 2011.

[2] Gentile, G. \& Noekel, K., Modelling Public Transport Passenger Flows in the Era of Intelligent Transport Systems: COST Action TU1004, Springer-Verlag: Berlin and New York, 2016.

[3] Friedrich, M. \& Wekeck, S., A schedule-based Transit Assignment Model addressing the Passengers' Choice among competing Connections. Schedule-Based Dynamic Transit Modeling: Theory and Applications, Kluwer Academic Publishers, pp. 159-173, 2004.

[4] Savelberg, F., Warffemius, P. \& Kroes, E., Estimation of the social cost of train delays in the Netherlands. Proceedings of the European Transport Conference, 2017.

[5] Noekel, K. \& Bundschuh, M., Robust transfers: transit timetable reliability from the passenger perspective. Proceedings of 3rd International Symposium on Transportation Network Reliability - INSTR, Den Haag, 2007

[6] Axhausen, K.W. et al., Ermittlung von Bewertungsansätzen für Reisezeiten und Zuverlässigkeit auf der Basis eines Modells für modale Verlagerungen im nichtgewerblichen und gewerblichen Personenverkehr für die Bundesverkehrswegeplanung, Bericht ETH Zürich, 2015.

[7] Fröhlich, P., Weis, C., Vrtic, M, Widmer, P. \& Aemisegger, P., Einfluss der Verlässlichkeit der Verkehrssysteme auf das Verkehrsverhalten, Schlussbericht SVI 2012/003, Schriftenreihe, 1472, UVEK, Ittigen, 2014.

[8] Franke, B. \& Lieberherr, J., Kombinierte betriebliche und verkehrliche Bewertung von Bahn-Angeboten. International Railway Symposium Aachen, 2017. 